\title{
EFFECTS OF SEISMIC ISOLATION ON TIME PERIOD AND STOREY SHEAR IN RC BUILDING WITH BRACINGS AND SHEAR WALLS
}

\author{
Raghunandan M H ${ }^{1}$, Suma Devi ${ }^{2}$ \\ ${ }^{1}$ Assistant Professor, Department of Civil Engineering, MSEC-Bengaluru, Karnataka, India \\ ${ }^{2}$ Assistant Professor, Department of Civil Engineering, SVCE-Bengaluru, Karnataka, India
}

\begin{abstract}
Higher the time period higher will be the flexibility this helps in controlling the structural damages during ground motions. In a flexible building different parts move by different amount, but for a stiff building every part moves by same amount. Depending on the situation either a flexible or a stiff structure can be made to work. In this study the comparison of fundamental time period and storey shear between fixed base building and isolated base building is done by modelled in Etabs non-linear software with different frame systems and storey height in order to know the variation of time period with different conditions.
\end{abstract}

Keywords: Fundamental time period, Storey shear, Bracings, Shear walls etc...

\section{INTRODUCTION}

In isolated structure due to insertion of a flexible layer between foundation and superstructure, the fundamental time-period of the structural system increases to a value higher than the predominant time-periods of earthquake ground motions. Since a base isolated structure has fundamental frequency lower than that of the frequency of its fixed base and as well as the predominant frequencies of ground motion, the first mode of vibration of the isolated structure involves deformation only in isolation system whereas the superstructure remains almost rigid. In this way, the isolation of base becomes an attractive and effective approach for protection of expensive equipments and internal non-structural components where needed (Kelly JM, 1986). The incorporation of seismic base isolation system aims at the avoidance of damage to the superstructure in contrast with the conventionally designed structures, where damages are very likely during strong earthquake.

\subsection{Materials and Methods}

The G+9 storied RC frame building is modelled in ETABS nonlinear software. The special moment resisting frame building is considered to be situated in zone $\mathrm{V}$ having medium soil type and intended for residential use. Beams and column members are modelled as frame elements, where the columns have been restrained in all six degrees of freedom at the base. Slabs are defined as area elements are defined as membrane elements and are modelled as rigid diaphragms. The concrete braces are defined as frame elements and shear walls as shell area elements.

Column size for building is $0.6 \times 1 \mathrm{~m}$, while the beam size is $0.35 \times 0.6 \mathrm{~m}$ and bracing size of $0.2 \times 0.2 \mathrm{~m}$, slab thickness of $130 \mathrm{~mm}$ and shear wall thickness of $200 \mathrm{~mm}$ are taken. Grade of concrete for beam, slab, brace and shear wall is taken as M20 and M25 for column, with unit weight of concrete as $25 \mathrm{kN} / \mathrm{m}^{3}$. The $\mathrm{G}+9$ storeys building will have 4 number of bays with $4 \mathrm{~m}$ width in $\mathrm{X}$ direction, 5 number of bays with $5 \mathrm{~m}$ width in $\mathrm{Y}$ direction, see Fig -1 and Fig -2 .

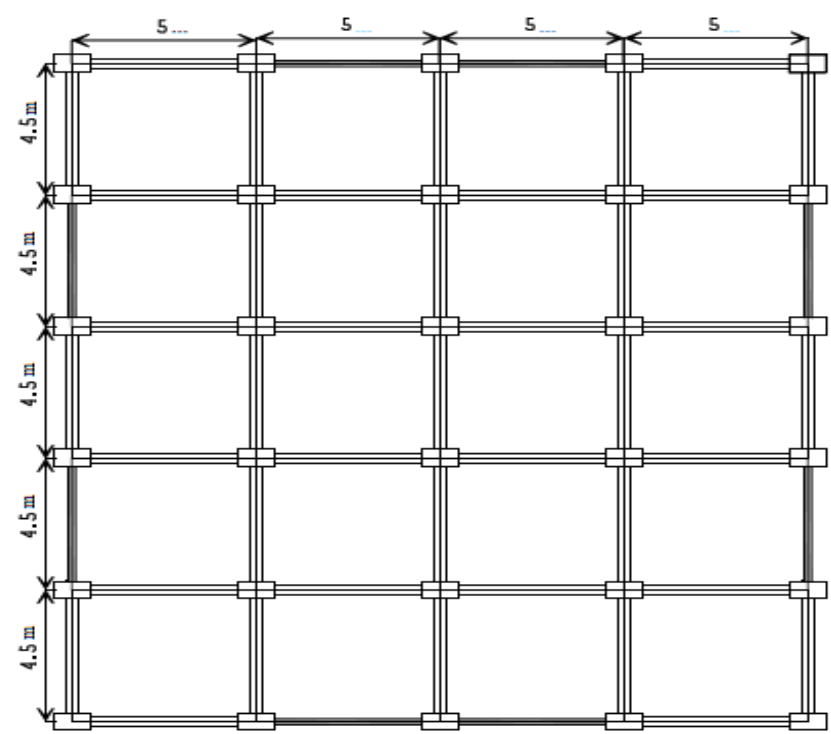

Fig -1: Building plan layout
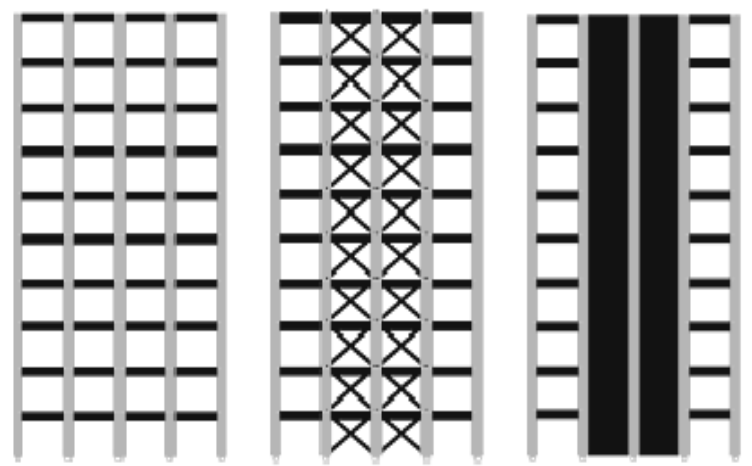

Fig -2: Elevation view of bare frame building, with Xbracings and with Shear Walls 


\section{RESULTS AND DISCUSSIONS}

\subsection{Fundamental Time Period}

It is the total time required for any structure to complete one cycle of vibration or a sway, which is expressed in second. In order to know the behavior of natural time period of various models the first mode time period of the models are obtained from equivalent static analysis in ETABS. Table-1, Chart-1A and Chart-1B shows the variation of time period for different conditions.

The time period value for base isolated structure is more when compared with base fixed structure; this is because the isolated structure moves without much restriction during earthquake so that the time taken by them to complete a cycle is high. In case of fixed base buildings, for every $0.2 \mathrm{~m}$ increase in storey height increases the time period value by $8-10 \%$, also for buildings with isolated base the time period increases by $2-3 \%$.

So we can say that Time period increases with the increase in storey height. The time period of fixed base buildings will reduce up to $20 \%$ by using $\mathrm{X}$-bracings and up to $35 \%$ by using shear walls. However in case of base isolated building by using $\mathrm{X}$ bracings or shear walls time period reduces only about 3\%. By this way the frequency of isolated base buildings will be least which helps in reducing the damages during the earthquake vibrations.

Table -1: Comparison of time period between fixed base and isolated Base buildings

\begin{tabular}{|ll|l|l|l|}
\hline \multirow{2}{*}{} & \multicolumn{4}{|l|}{ Time Period (second) } \\
\cline { 2 - 5 } & \multicolumn{4}{|l|}{ Fixed base G+9 storey building } \\
\hline $\begin{array}{l}\text { Storey } \\
(\mathbf{m})\end{array}$ & ht. & Bare frame & $\begin{array}{l}\text { With } \\
\text { Bracing }\end{array}$ & $\begin{array}{l}\text { With } \\
\text { Shearwall }\end{array}$ \\
\hline 2.8 & & 1.11619 & 0.88597 & 0.66208 \\
\hline 3 & 1.21735 & 0.95645 & 0.72581 \\
\hline 3.2 & 1.3212 & 1.02901 & 0.79172 \\
\hline & & \multicolumn{2}{|l}{ Isolated base G+9 storey building } \\
\hline $\begin{array}{l}\text { Storey } \\
(\mathbf{m})\end{array}$ & ht. & $\begin{array}{l}\text { Isolated } \\
\text { Bare frame }\end{array}$ & $\begin{array}{l}\text { Isolated } \\
\text { with } \\
\text { Bracing }\end{array}$ & $\begin{array}{l}\text { Isolated } \\
\text { with } \\
\text { Shearwall }\end{array}$ \\
\hline 2.8 & & 2.68541 & 2.62819 & 2.64831 \\
\hline 3 & 2.74733 & 2.6736 & 2.69621 \\
\hline 3.2 & 2.81306 & 2.72066 & 2.74513 \\
\hline
\end{tabular}

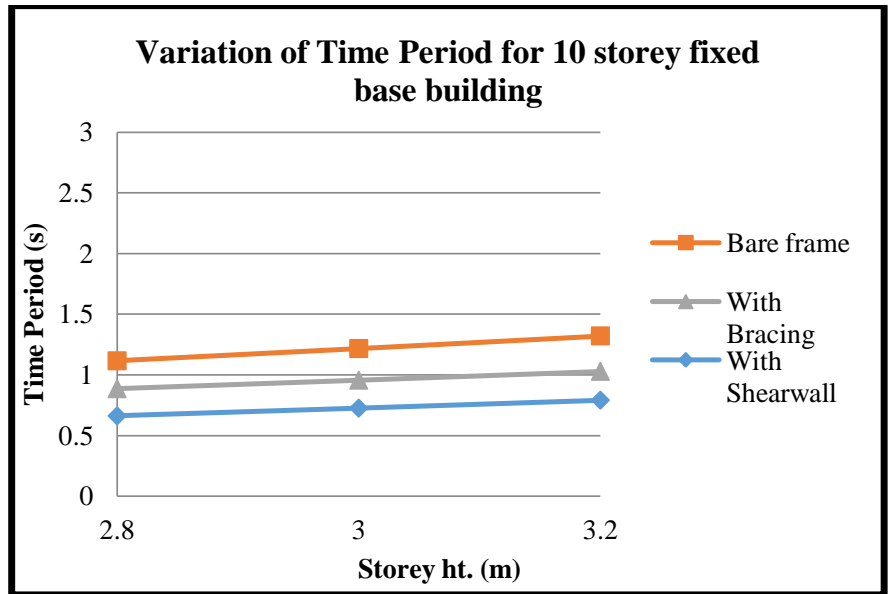

Chart -1 A: Variation of Time period for G+9 storey fixed base building

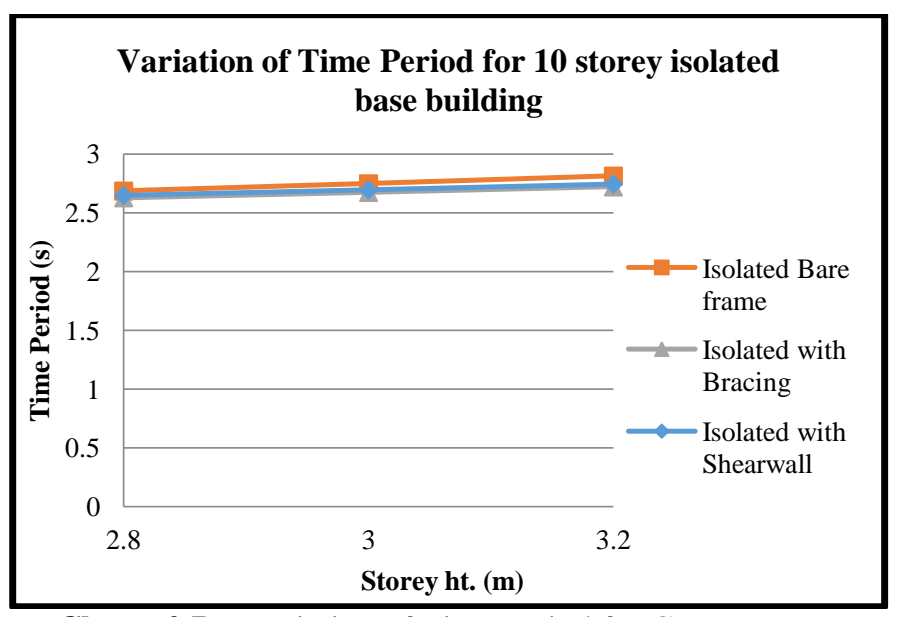

Chart -2 B: Variation of Time period for G+9 storey isolated base building

\subsection{Storey Shear}

The storey shear at each storey level for various building model in both $\mathrm{X}$ and $\mathrm{Y}$ directions are presented in Table-2 and Table-3, which are obtained from the equivalent static analysis and can be visualized in Chart-2 and Chart-3. By observing the below tables and charts we see that for every $0.2 \mathrm{~m}$ increase in storey height reduces the storey shear by 5 $10 \%$ in fixed base building and $1-2 \%$ in case of isolated base building. This indicates that storey shear decreases with increase in storey height. Also in case of fixed base buildings the storey shear increases by $10-30 \%$ with addition of bracings and $30-50 \%$ with addition of shearwalls. This shows that storey shear increases by use of bracings or shearwalls.

However in isolated base buildings the storey shear increases only by $5-15 \%$ with addition of bracings or shearwalls. Therefore we can say that by varying the storey height and addition of lateral load resisting systems the storey shear value can varies in case of fixed base buildings than isolated base buildings that has least storey shear value. 
Table -2: Storey shear of fixed base building with variation of storey height and frame system

\begin{tabular}{|c|c|c|c|c|c|c|}
\hline \multirow{3}{*}{ ESA } & \multicolumn{6}{|c|}{ Storey shear of fixed base building (kN) } \\
\hline & \multicolumn{2}{|c|}{$\begin{array}{l}\text { BARE } \\
\text { FRAME }\end{array}$} & \multicolumn{2}{|c|}{$\begin{array}{l}\text { WITH } \\
\text { BRACING }\end{array}$} & \multicolumn{2}{|c|}{$\begin{array}{l}\text { WITH } \\
\text { SHEAEWAL } \\
\text { L }\end{array}$} \\
\hline & VX & VY & VX & VY & VX & VY \\
\hline $\begin{array}{l}\text { Storey } \\
\text { no }\end{array}$ & $\begin{array}{l}2.8 \mathrm{~m} \\
\text { height }\end{array}$ & storey & $\begin{array}{l}2.8 m \\
\text { height }\end{array}$ & storey & $\begin{array}{l}2.8 m \\
\text { height }\end{array}$ & storey \\
\hline 10 & $\begin{array}{l}804.0 \\
8\end{array}$ & 765.5 & $\begin{array}{l}1044 . \\
1\end{array}$ & $\begin{array}{l}977.9 \\
6\end{array}$ & 1629 & $\begin{array}{l}1338 . \\
5\end{array}$ \\
\hline 9 & $\begin{array}{l}1591 . \\
7\end{array}$ & $\begin{array}{l}1515 . \\
4\end{array}$ & $\begin{array}{l}2075 . \\
3\end{array}$ & $\begin{array}{l}1943 . \\
9\end{array}$ & $\begin{array}{l}3316 . \\
1\end{array}$ & $\begin{array}{l}2724 . \\
7\end{array}$ \\
\hline 8 & 2214 & $\begin{array}{l}2107 . \\
8\end{array}$ & $\begin{array}{l}2890 . \\
1\end{array}$ & 2707 & $\begin{array}{l}4649 . \\
2\end{array}$ & 3820 \\
\hline 7 & $\begin{array}{l}2690 . \\
5\end{array}$ & $\begin{array}{l}2561 . \\
4\end{array}$ & $\begin{array}{l}3513 . \\
9\end{array}$ & $\begin{array}{l}3291 . \\
3\end{array}$ & $\begin{array}{l}5669 . \\
8\end{array}$ & $\begin{array}{l}4658 . \\
6 \\
\end{array}$ \\
\hline 6 & $\begin{array}{l}3040 . \\
6\end{array}$ & $\begin{array}{l}2894 . \\
7\end{array}$ & $\begin{array}{l}3972 . \\
2\end{array}$ & $\begin{array}{l}3720 . \\
6\end{array}$ & $\begin{array}{l}6419 . \\
6\end{array}$ & $\begin{array}{l}5274 . \\
7\end{array}$ \\
\hline 5 & $\begin{array}{l}3283 . \\
7\end{array}$ & $\begin{array}{l}3126 . \\
1\end{array}$ & $\begin{array}{l}4290 . \\
5\end{array}$ & $\begin{array}{l}4018 . \\
8\end{array}$ & $\begin{array}{l}6940 . \\
3\end{array}$ & $\begin{array}{l}5702 . \\
6\end{array}$ \\
\hline 4 & $\begin{array}{l}3439 . \\
2\end{array}$ & $\begin{array}{l}3274 . \\
3\end{array}$ & $\begin{array}{l}4494 . \\
2\end{array}$ & $\begin{array}{l}4209 . \\
5\end{array}$ & $\begin{array}{l}7273 . \\
6 \\
\end{array}$ & $\begin{array}{l}5976 . \\
4\end{array}$ \\
\hline 3 & $\begin{array}{l}3526 . \\
8\end{array}$ & $\begin{array}{l}3357 . \\
6\end{array}$ & $\begin{array}{l}4608 . \\
7\end{array}$ & $\begin{array}{l}4316 . \\
9\end{array}$ & 7461 & $\begin{array}{l}6130 . \\
4\end{array}$ \\
\hline 2 & $\begin{array}{l}3565 . \\
7\end{array}$ & $\begin{array}{l}3394 . \\
6\end{array}$ & $\begin{array}{l}4659 . \\
7\end{array}$ & $\begin{array}{l}4364 . \\
6\end{array}$ & $\begin{array}{l}7544 . \\
4\end{array}$ & $\begin{array}{l}6198 . \\
9\end{array}$ \\
\hline 1 & $\begin{array}{l}3575 . \\
4\end{array}$ & $\begin{array}{l}3403 . \\
9\end{array}$ & $\begin{array}{l}4672 . \\
4\end{array}$ & $\begin{array}{l}4376 . \\
5\end{array}$ & $\begin{array}{l}7565 . \\
2\end{array}$ & 6216 \\
\hline BASE & $\begin{array}{l}3575 . \\
4\end{array}$ & $\begin{array}{l}3403 . \\
9\end{array}$ & $\begin{array}{l}4672 . \\
4\end{array}$ & $\begin{array}{l}4376 . \\
5\end{array}$ & $\begin{array}{l}7565 . \\
2\end{array}$ & 6216 \\
\hline
\end{tabular}

\begin{tabular}{|c|c|c|c|c|c|c|}
\hline $\begin{array}{l}\text { Storey } \\
\text { no }\end{array}$ & \multicolumn{2}{|c|}{$\begin{array}{l}\text { 3.0m storey } \\
\text { height }\end{array}$} & \multicolumn{2}{|c|}{$\begin{array}{l}\text { 3.0m storey } \\
\text { height }\end{array}$} & \multicolumn{2}{|c|}{$\begin{array}{l}\text { 3.0m storey } \\
\text { height }\end{array}$} \\
\hline 10 & 748.4 & $\begin{array}{l}711.9 \\
4\end{array}$ & $\begin{array}{l}985.4 \\
7\end{array}$ & $\begin{array}{l}918.8 \\
9\end{array}$ & $\begin{array}{l}1600 . \\
8\end{array}$ & $\begin{array}{l}1244 . \\
7\end{array}$ \\
\hline 9 & $\begin{array}{l}1487 . \\
7\end{array}$ & $\begin{array}{l}1415 . \\
2\end{array}$ & $\begin{array}{l}1966 . \\
9\end{array}$ & 1834 & $\begin{array}{l}3275 . \\
4\end{array}$ & $\begin{array}{l}2546 . \\
8\end{array}$ \\
\hline 8 & $\begin{array}{l}2071 . \\
8\end{array}$ & $\begin{array}{l}1970 . \\
9\end{array}$ & $\begin{array}{l}2742 . \\
4\end{array}$ & $\begin{array}{l}2557 . \\
1\end{array}$ & $\begin{array}{l}4598 . \\
5\end{array}$ & $\begin{array}{l}3575 . \\
6\end{array}$ \\
\hline 7 & $\begin{array}{l}2519 . \\
1\end{array}$ & $\begin{array}{l}2396 . \\
3\end{array}$ & $\begin{array}{l}3336 . \\
1\end{array}$ & $\begin{array}{l}3110 . \\
7\end{array}$ & $\begin{array}{l}5611 . \\
5\end{array}$ & $\begin{array}{l}4363 . \\
3\end{array}$ \\
\hline 6 & $\begin{array}{l}2847 . \\
6\end{array}$ & $\begin{array}{l}2708 . \\
9\end{array}$ & $\begin{array}{l}3772 . \\
3\end{array}$ & $\begin{array}{l}3517 . \\
4\end{array}$ & $\begin{array}{l}6355 . \\
8\end{array}$ & 4942 \\
\hline 5 & $\begin{array}{l}3075 . \\
8\end{array}$ & $\begin{array}{l}2925 . \\
9\end{array}$ & $\begin{array}{l}4075 . \\
2\end{array}$ & $\begin{array}{l}3799 . \\
8\end{array}$ & $\begin{array}{l}6872 . \\
6 \\
\end{array}$ & $\begin{array}{l}5343 . \\
9\end{array}$ \\
\hline 4 & $\begin{array}{l}3221 . \\
8 \\
\end{array}$ & $\begin{array}{l}3064 . \\
9\end{array}$ & 4269 & $\begin{array}{l}3980 . \\
6 \\
\end{array}$ & $\begin{array}{l}7203 . \\
4\end{array}$ & $\begin{array}{l}5601 . \\
1\end{array}$ \\
\hline 3 & 3304 & 3143 & $\begin{array}{l}4378 . \\
1\end{array}$ & $\begin{array}{l}4082 . \\
3\end{array}$ & $\begin{array}{l}7389 . \\
5\end{array}$ & $\begin{array}{l}5745 . \\
7\end{array}$ \\
\hline 2 & $\begin{array}{l}3340 . \\
5 \\
\end{array}$ & $\begin{array}{l}3177 . \\
7 \\
\end{array}$ & $\begin{array}{l}4426 . \\
5 \\
\end{array}$ & $\begin{array}{l}4127 . \\
4\end{array}$ & $\begin{array}{l}7472 . \\
2 \\
\end{array}$ & 5810 \\
\hline 1 & $\begin{array}{l}3349 . \\
6 \\
\end{array}$ & $\begin{array}{l}3186 . \\
4\end{array}$ & $\begin{array}{l}4438 . \\
7 \\
\end{array}$ & $\begin{array}{l}4138 . \\
7\end{array}$ & $\begin{array}{l}7492 . \\
8 \\
\end{array}$ & $\begin{array}{l}5826 . \\
1 \\
\end{array}$ \\
\hline BASE & $\begin{array}{l}3349 . \\
6\end{array}$ & $\begin{array}{l}3186 . \\
4\end{array}$ & $\begin{array}{l}4438 . \\
7\end{array}$ & $\begin{array}{l}4138 . \\
7\end{array}$ & $\begin{array}{l}7492 . \\
8\end{array}$ & $\begin{array}{l}5826 . \\
1\end{array}$ \\
\hline
\end{tabular}

\begin{tabular}{|c|c|c|c|c|c|c|}
\hline $\begin{array}{l}\text { Storey } \\
\text { no }\end{array}$ & \multicolumn{2}{|c|}{$\begin{array}{l}3.2 \mathrm{~m} \text { storey } \\
\text { height }\end{array}$} & \multicolumn{2}{|c|}{$\begin{array}{l}3.2 \mathrm{~m} \text { storey } \\
\text { height }\end{array}$} & \multicolumn{2}{|c|}{$\begin{array}{l}3.2 \mathrm{~m} \text { storey } \\
\text { height }\end{array}$} \\
\hline 10 & $\begin{array}{l}699.9 \\
6\end{array}$ & $\begin{array}{l}665.2 \\
1\end{array}$ & $\begin{array}{l}933.0 \\
9\end{array}$ & $\begin{array}{l}866.1 \\
7\end{array}$ & $\begin{array}{l}1496 . \\
5\end{array}$ & $\begin{array}{l}1162 . \\
9\end{array}$ \\
\hline 9 & $\begin{array}{l}1397 . \\
1\end{array}$ & $\begin{array}{l}1327 . \\
7\end{array}$ & $\begin{array}{l}1869 . \\
8\end{array}$ & $\begin{array}{l}1735 . \\
7\end{array}$ & 3077 & 2391 \\
\hline 8 & $\begin{array}{l}1947 . \\
8\end{array}$ & $\begin{array}{l}1851 . \\
2\end{array}$ & 2610 & $\begin{array}{l}2422 . \\
8 \\
\end{array}$ & $\begin{array}{l}4325 . \\
8 \\
\end{array}$ & $\begin{array}{l}3361 . \\
4\end{array}$ \\
\hline 7 & $\begin{array}{l}2369 . \\
5\end{array}$ & $\begin{array}{l}2251 . \\
9\end{array}$ & $\begin{array}{l}3176 . \\
7\end{array}$ & $\begin{array}{l}2948 . \\
8\end{array}$ & $\begin{array}{l}5281 . \\
9\end{array}$ & $\begin{array}{l}4104 . \\
4\end{array}$ \\
\hline 6 & $\begin{array}{l}2679 . \\
4\end{array}$ & $\begin{array}{l}2546 . \\
4\end{array}$ & 3593 & $\begin{array}{l}3335 . \\
3\end{array}$ & $\begin{array}{l}5984 . \\
3 \\
\end{array}$ & $\begin{array}{l}4650 . \\
2\end{array}$ \\
\hline 5 & $\begin{array}{l}2894 . \\
5\end{array}$ & $\begin{array}{l}2750 . \\
8\end{array}$ & $\begin{array}{l}3882 . \\
1\end{array}$ & $\begin{array}{l}3603 . \\
7\end{array}$ & $\begin{array}{l}6472 . \\
1\end{array}$ & $\begin{array}{l}5029 . \\
3\end{array}$ \\
\hline 4 & $\begin{array}{l}3032 . \\
2\end{array}$ & $\begin{array}{l}2881 . \\
7\end{array}$ & $\begin{array}{l}4067 . \\
2\end{array}$ & $\begin{array}{l}3775 . \\
5\end{array}$ & $\begin{array}{l}6784 . \\
3 \\
\end{array}$ & $\begin{array}{l}5271 . \\
9\end{array}$ \\
\hline 3 & $\begin{array}{l}3109 . \\
7 \\
\end{array}$ & $\begin{array}{l}2955 . \\
3 \\
\end{array}$ & $\begin{array}{l}4171 . \\
2 \\
\end{array}$ & $\begin{array}{l}3872 . \\
1 \\
\end{array}$ & $\begin{array}{l}6959 . \\
9 \\
\end{array}$ & $\begin{array}{l}5408 . \\
3 \\
\end{array}$ \\
\hline 2 & $\begin{array}{l}3144 . \\
1\end{array}$ & 2988 & $\begin{array}{l}4217 . \\
5\end{array}$ & 3915 & 7038 & 5469 \\
\hline 1 & $\begin{array}{l}3152 . \\
7\end{array}$ & $\begin{array}{l}2996 . \\
2\end{array}$ & $\begin{array}{l}4229 . \\
1\end{array}$ & $\begin{array}{l}3925 . \\
8 \\
\end{array}$ & $\begin{array}{l}7057 . \\
5\end{array}$ & $\begin{array}{l}5484 . \\
1 \\
\end{array}$ \\
\hline BASE & $\begin{array}{l}3152 . \\
7\end{array}$ & $\begin{array}{l}2996 . \\
2\end{array}$ & $\begin{array}{l}4229 . \\
1\end{array}$ & $\begin{array}{l}3925 . \\
8\end{array}$ & $\begin{array}{l}7057 . \\
5\end{array}$ & $\begin{array}{l}5484 . \\
1\end{array}$ \\
\hline
\end{tabular}
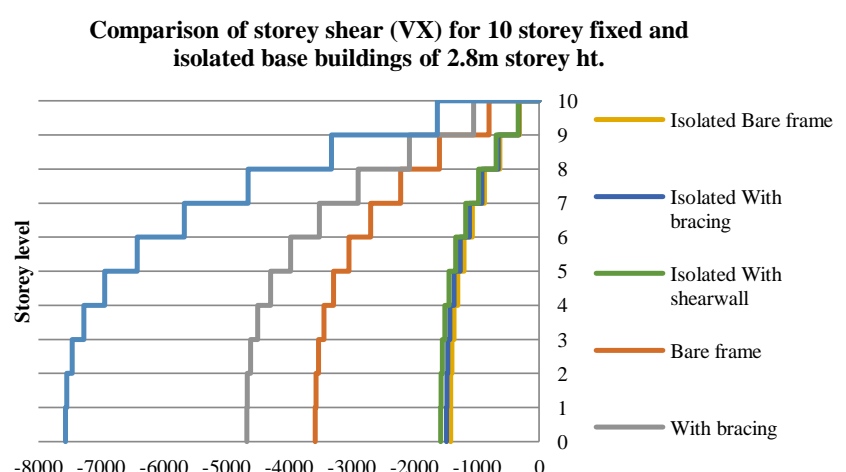

Storey shear in $\mathrm{X}$ direction $(\mathrm{kN})$

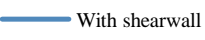

Comparison of storey shear (VX) for 10 storey fixed and isolated base buildings of $3.0 \mathrm{~m}$ storey ht.

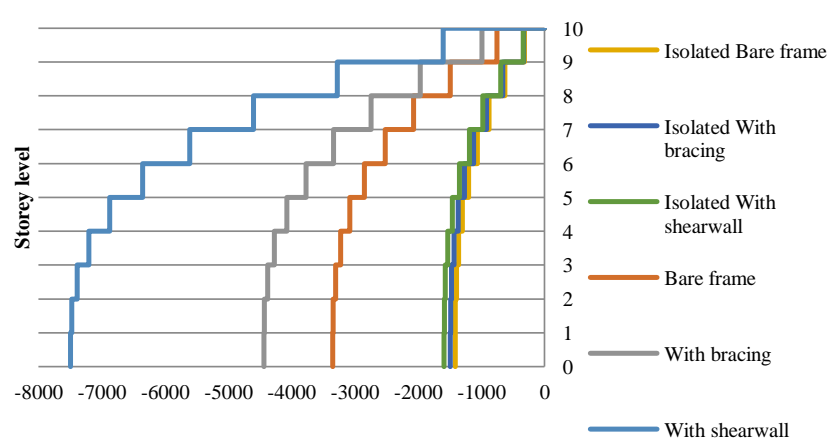

Storey shear in $\mathrm{X}$ direction $(\mathrm{kN})$ 


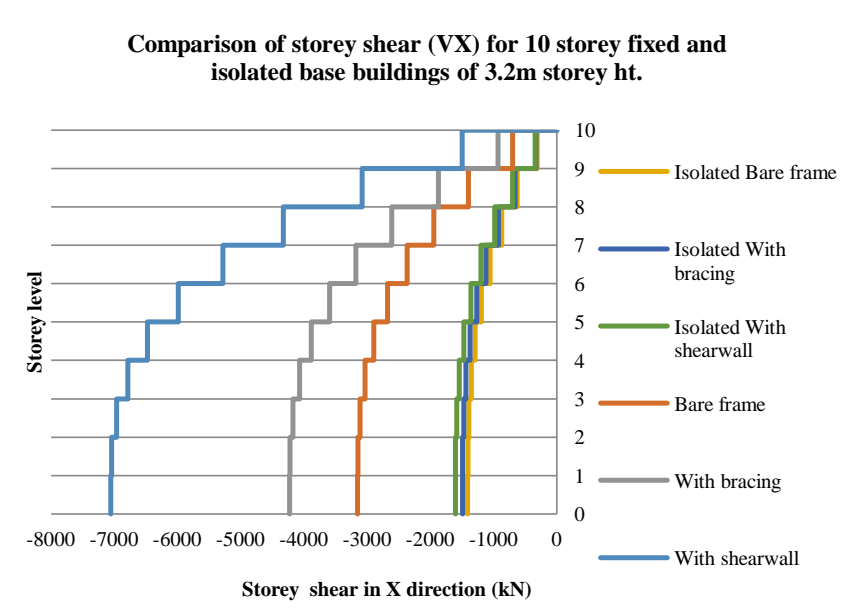

Chart -2: Comparison of Storey shear in X direction between fixed base and isolated base buildings

Table -3: Storey shear of isolated base building with variation of storey height and frame system

\begin{tabular}{|c|c|c|c|c|c|c|}
\hline \multirow{3}{*}{ ESA } & \multicolumn{6}{|c|}{ Storey shear of isolated base building (kN) } \\
\hline & \multicolumn{2}{|c|}{$\begin{array}{l}\text { BARE } \\
\text { FRAME }\end{array}$} & \multicolumn{2}{|c|}{$\begin{array}{l}\text { WITH } \\
\text { BRACING }\end{array}$} & \multicolumn{2}{|c|}{$\begin{array}{l}\text { WITH } \\
\text { SHEAEWAL } \\
\text { L }\end{array}$} \\
\hline & $\mathbf{V X}$ & VY & $\mathbf{V X}$ & VY & $\mathbf{V X}$ & VY \\
\hline $\begin{array}{l}\text { Storey } \\
\text { no }\end{array}$ & $\begin{array}{l}2.8 m \\
\text { height }\end{array}$ & storey & $\begin{array}{l}2.8 m \\
\text { height }\end{array}$ & storey & $\begin{array}{l}2.8 m \\
\text { height }\end{array}$ & storey \\
\hline 10 & $\begin{array}{l}318.1 \\
8\end{array}$ & $\begin{array}{l}318.2 \\
3\end{array}$ & $\begin{array}{l}330.7 \\
9\end{array}$ & $\begin{array}{l}329.6 \\
7\end{array}$ & $\begin{array}{l}338.1 \\
4\end{array}$ & $\begin{array}{l}334.6 \\
1\end{array}$ \\
\hline 9 & $\begin{array}{l}629.8 \\
6\end{array}$ & $\begin{array}{l}629.9 \\
5\end{array}$ & $\begin{array}{l}657.4 \\
9\end{array}$ & $\begin{array}{l}655.2 \\
8\end{array}$ & $\begin{array}{l}688.3 \\
6\end{array}$ & $\begin{array}{l}681.1 \\
7\end{array}$ \\
\hline 8 & $\begin{array}{l}876.1 \\
2\end{array}$ & $\begin{array}{l}876.2 \\
5\end{array}$ & $\begin{array}{l}915.6 \\
3\end{array}$ & $\begin{array}{l}912.5 \\
4\end{array}$ & $\begin{array}{l}965.0 \\
7\end{array}$ & 955 \\
\hline 7 & $\begin{array}{l}1064 . \\
7\end{array}$ & $\begin{array}{l}1064 . \\
8\end{array}$ & $\begin{array}{l}1113 . \\
3\end{array}$ & $\begin{array}{l}1109 . \\
5\end{array}$ & $\begin{array}{l}1176 . \\
9\end{array}$ & $\begin{array}{l}1164 . \\
7\end{array}$ \\
\hline 6 & $\begin{array}{l}1203 . \\
2\end{array}$ & $\begin{array}{l}1203 . \\
4\end{array}$ & $\begin{array}{l}1258 . \\
5\end{array}$ & $\begin{array}{l}1254 . \\
2\end{array}$ & $\begin{array}{l}1332 . \\
6 \\
\end{array}$ & $\begin{array}{l}1318 . \\
7\end{array}$ \\
\hline 5 & $\begin{array}{l}1299 . \\
4\end{array}$ & $\begin{array}{l}1299 . \\
6\end{array}$ & $\begin{array}{l}1359 . \\
3\end{array}$ & $\begin{array}{l}1354 . \\
7\end{array}$ & $\begin{array}{l}1440 . \\
7\end{array}$ & $\begin{array}{l}1425 . \\
6\end{array}$ \\
\hline 4 & 1361 & $\begin{array}{l}1361 . \\
2\end{array}$ & $\begin{array}{l}1423 . \\
9\end{array}$ & 1419 & $\begin{array}{l}1509 . \\
9\end{array}$ & $\begin{array}{l}1494 . \\
1\end{array}$ \\
\hline 3 & $\begin{array}{l}1395 . \\
6\end{array}$ & $\begin{array}{l}1395 . \\
8\end{array}$ & $\begin{array}{l}1460 . \\
2\end{array}$ & $\begin{array}{l}1455 . \\
2\end{array}$ & $\begin{array}{l}1548 . \\
8\end{array}$ & $\begin{array}{l}1532 . \\
6\end{array}$ \\
\hline 2 & 1411 & $\begin{array}{l}1411 . \\
2\end{array}$ & $\begin{array}{l}1476 . \\
3\end{array}$ & $\begin{array}{l}1471 . \\
3\end{array}$ & $\begin{array}{l}1566 . \\
1\end{array}$ & $\begin{array}{l}1549 . \\
7\end{array}$ \\
\hline 1 & $\begin{array}{l}1414 . \\
8\end{array}$ & 1415 & $\begin{array}{l}1480 . \\
3\end{array}$ & $\begin{array}{l}1475 . \\
3\end{array}$ & $\begin{array}{l}1570 . \\
4\end{array}$ & 1554 \\
\hline BASE & $\begin{array}{l}1414 . \\
8\end{array}$ & 1415 & $\begin{array}{l}1480 . \\
3\end{array}$ & $\begin{array}{l}1475 . \\
3\end{array}$ & $\begin{array}{l}1570 . \\
4\end{array}$ & 1554 \\
\hline
\end{tabular}

\begin{tabular}{|l|l|l|l|l|l|l|}
\hline $\begin{array}{l}\text { Storey } \\
\text { no }\end{array}$ & \multicolumn{2}{l}{$\begin{array}{l}\text { 3.0m } \\
\text { height }\end{array}$} & \multicolumn{2}{l|}{$\begin{array}{l}\text { 3.0m } \\
\text { height }\end{array}$} & \multicolumn{2}{l|}{$\begin{array}{l}\text { 3.0m storey } \\
\text { height }\end{array}$} \\
\hline \multirow{2}{*}{10} & 315.5 & 315.4 & 330.2 & 328.7 & 339.1 & 335.0 \\
& 7 & 6 & 1 & 2 & 9 & 7 \\
\hline 9 & 627.2 & 627.0 & 659.0 & 656.0 & 694.0 & 685.5 \\
& 9 & 8 & 7 & 9 & 1 & 9 \\
\hline \multirow{2}{*}{8} & 873.5 & 873.3 & 918.9 & 914.7 & 974.3 & 962.5 \\
& 9 & & 6 & 6 & 4 \\
\hline 7 & 1062. & 1061. & 1117. & 1112. & 1189 & 1174. \\
\hline
\end{tabular}

\begin{tabular}{|c|c|c|c|c|c|c|}
\hline & 2 & 8 & 9 & 8 & & 6 \\
\hline 6 & $\begin{array}{l}1200 . \\
7\end{array}$ & $\begin{array}{l}1200 . \\
3\end{array}$ & 1264 & $\begin{array}{l}1258 . \\
3\end{array}$ & $\begin{array}{l}1346 . \\
7\end{array}$ & $\begin{array}{l}1330 . \\
4\end{array}$ \\
\hline 5 & $\begin{array}{l}1296 . \\
9\end{array}$ & $\begin{array}{l}1296 . \\
5\end{array}$ & $\begin{array}{l}1365 . \\
5\end{array}$ & $\begin{array}{l}1359 . \\
3\end{array}$ & $\begin{array}{l}1456 . \\
2\end{array}$ & $\begin{array}{l}1438 . \\
6\end{array}$ \\
\hline 4 & $\begin{array}{l}1358 . \\
5\end{array}$ & 1358 & $\begin{array}{l}1430 . \\
5\end{array}$ & 1424 & $\begin{array}{l}1526 . \\
3\end{array}$ & $\begin{array}{l}1507 . \\
8\end{array}$ \\
\hline 3 & $\begin{array}{l}1393 . \\
1\end{array}$ & $\begin{array}{l}1392 . \\
7\end{array}$ & 1467 & $\begin{array}{l}1460 . \\
4\end{array}$ & $\begin{array}{l}1565 . \\
7\end{array}$ & $\begin{array}{l}1546 . \\
7\end{array}$ \\
\hline 2 & $\begin{array}{l}1408 . \\
5\end{array}$ & $\begin{array}{l}1408 . \\
1\end{array}$ & $\begin{array}{l}1483 . \\
3\end{array}$ & $\begin{array}{l}1476 . \\
5\end{array}$ & $\begin{array}{l}1583 . \\
3\end{array}$ & $\begin{array}{l}1564 . \\
1\end{array}$ \\
\hline 1 & $\begin{array}{l}1412 . \\
4\end{array}$ & $\begin{array}{l}1411 . \\
9\end{array}$ & $\begin{array}{l}1487 . \\
3\end{array}$ & $\begin{array}{l}1480 . \\
6\end{array}$ & $\begin{array}{l}1587 . \\
6\end{array}$ & $\begin{array}{l}1568 . \\
4\end{array}$ \\
\hline BASE & $\begin{array}{l}1412 . \\
4\end{array}$ & $\begin{array}{l}1411 . \\
9\end{array}$ & $\begin{array}{l}1487 . \\
3\end{array}$ & $\begin{array}{l}1480 . \\
6\end{array}$ & $\begin{array}{l}1587 . \\
6\end{array}$ & $\begin{array}{l}1568 . \\
4\end{array}$ \\
\hline
\end{tabular}

\begin{tabular}{|c|c|c|c|c|c|c|}
\hline $\begin{array}{l}\text { Storey } \\
\text { no }\end{array}$ & $\begin{array}{l}3.2 \mathrm{~m} \\
\text { height }\end{array}$ & storey & $\begin{array}{l}3.2 \mathrm{~m} \\
\text { height }\end{array}$ & storey & $\begin{array}{l}3.2 \mathrm{~m} \\
\text { height }\end{array}$ & storey \\
\hline 10 & $\begin{array}{l}312.7 \\
3\end{array}$ & $\begin{array}{l}312.4 \\
3\end{array}$ & $\begin{array}{l}329.5 \\
2\end{array}$ & $\begin{array}{l}327.6 \\
1\end{array}$ & $\begin{array}{l}340.1 \\
3\end{array}$ & $\begin{array}{l}335.3 \\
8\end{array}$ \\
\hline 9 & $\begin{array}{l}624.1 \\
7\end{array}$ & $\begin{array}{l}623.5 \\
8\end{array}$ & $\begin{array}{l}660.3 \\
3\end{array}$ & 656.5 & $\begin{array}{l}699.3 \\
5\end{array}$ & $\begin{array}{l}689.5 \\
9\end{array}$ \\
\hline 8 & $\begin{array}{l}870.2 \\
5\end{array}$ & $\begin{array}{l}869.4 \\
2\end{array}$ & $\begin{array}{l}921.7 \\
2\end{array}$ & $\begin{array}{l}916.3 \\
6\end{array}$ & $\begin{array}{l}983.1 \\
8\end{array}$ & $\begin{array}{l}969.4 \\
6\end{array}$ \\
\hline 7 & $\begin{array}{l}1058 . \\
7\end{array}$ & $\begin{array}{l}1057 . \\
7\end{array}$ & $\begin{array}{l}1121 . \\
8\end{array}$ & $\begin{array}{l}1115 . \\
3\end{array}$ & $\begin{array}{l}1200 . \\
5\end{array}$ & $\begin{array}{l}1183 . \\
7\end{array}$ \\
\hline 6 & $\begin{array}{l}1197 . \\
1\end{array}$ & $\begin{array}{l}1195 . \\
9\end{array}$ & $\begin{array}{l}1268 . \\
9\end{array}$ & $\begin{array}{l}1261 . \\
5\end{array}$ & $\begin{array}{l}1360 . \\
2\end{array}$ & $\begin{array}{l}1341 . \\
2\end{array}$ \\
\hline 5 & $\begin{array}{l}1293 . \\
2\end{array}$ & 1292 & 1371 & 1363 & 1471 & $\begin{array}{l}1450 . \\
5\end{array}$ \\
\hline 4 & $\begin{array}{l}1354 . \\
7\end{array}$ & $\begin{array}{l}1353 . \\
4\end{array}$ & $\begin{array}{l}1436 . \\
3\end{array}$ & 1428 & 1542 & $\begin{array}{l}1520 . \\
5\end{array}$ \\
\hline 3 & $\begin{array}{l}1389 . \\
3\end{array}$ & 1388 & $\begin{array}{l}1473 . \\
1\end{array}$ & $\begin{array}{l}1464 . \\
5\end{array}$ & $\begin{array}{l}1581 . \\
9\end{array}$ & $\begin{array}{l}1559 . \\
8\end{array}$ \\
\hline 2 & $\begin{array}{l}1404 . \\
7\end{array}$ & $\begin{array}{l}1403 . \\
4\end{array}$ & $\begin{array}{l}1489 . \\
4\end{array}$ & $\begin{array}{l}1480 . \\
8\end{array}$ & $\begin{array}{l}1599 . \\
6\end{array}$ & $\begin{array}{l}1577 . \\
3\end{array}$ \\
\hline 1 & $\begin{array}{l}1408 . \\
6\end{array}$ & $\begin{array}{l}1407 . \\
2\end{array}$ & $\begin{array}{l}1493 . \\
5\end{array}$ & $\begin{array}{l}1484 . \\
8\end{array}$ & $\begin{array}{l}1604 . \\
1\end{array}$ & $\begin{array}{l}1581 . \\
7\end{array}$ \\
\hline BASE & $\begin{array}{l}1408 . \\
6\end{array}$ & $\begin{array}{l}1407 . \\
2\end{array}$ & $\begin{array}{l}1493 . \\
5\end{array}$ & $\begin{array}{l}1484 . \\
8\end{array}$ & $\begin{array}{l}1604 . \\
1\end{array}$ & $\begin{array}{l}1581 . \\
7\end{array}$ \\
\hline
\end{tabular}

Comparison of storey shear (VY) for 10 storey fixed and isolated base buildings of $2.8 \mathrm{~m}$ storey ht.

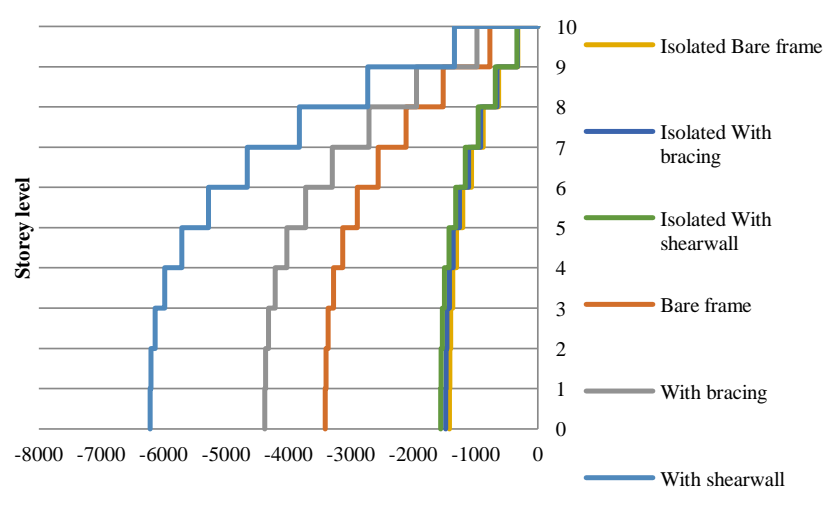

Storey shear in $\mathrm{Y}$ direction (kN) 
Comparison of storey shear (VY) for 10 storey fixed and isolated base buildings of $3.0 \mathrm{~m}$ storey ht.

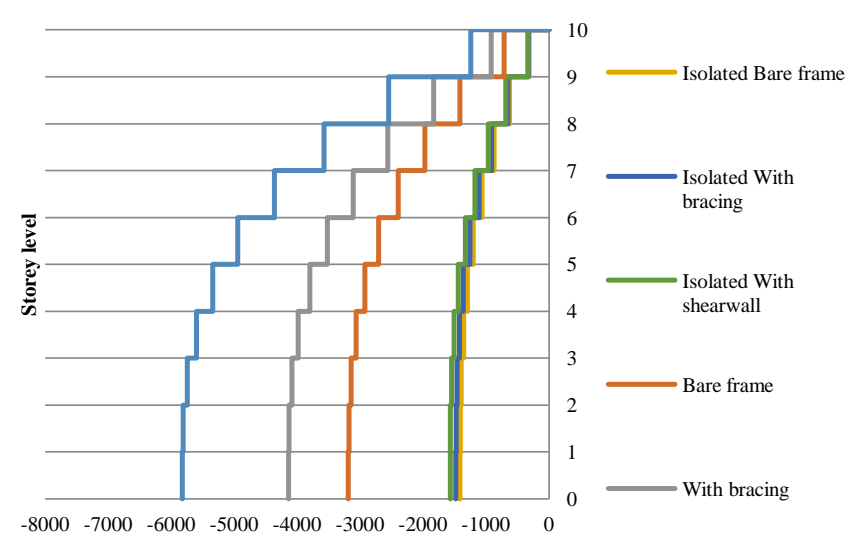

Storey shear in $\mathrm{Y}$ direction $(\mathrm{kN})$

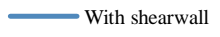

Comparison of storey shear (VY) for 10 storey fixed and isolated base buildings of $3.2 \mathrm{~m}$ storey ht.

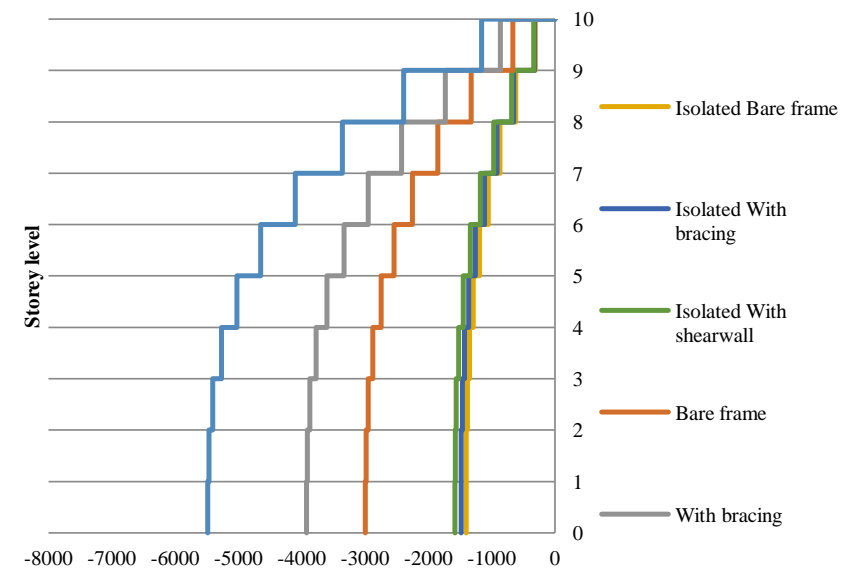

Storey shear in $\mathrm{Y}$ direction $(\mathrm{kN}) \quad$ With shearwall

Chart -3: Comparison of Storey shear in Y direction between fixed base and isolated base buildings

\section{CONCLUSION}

\subsection{Fundamental Time Period}

- Time period increases with the increase in storey height, which is more sensitive in fixed base rather than isolated base building.

- $\quad$ Also the time period of the fixed base buildings will reduce up to $20 \%$ by using $\mathrm{X}$-bracings and up to $35 \%$ by using shear walls.

- However in case of base isolated building by using $\mathrm{X}$ bracings or shear walls the time period reduces only about $3 \%$.

- $\quad$ The time period value for base isolated structure is more when compared with base fixed structure. This helps in reducing the damages due to earthquake vibrations.

\subsection{Storey Shear}

- For every $0.2 \mathrm{~m}$ increase in storey height reduces the storey shear by $5-10 \%$ in fixed base building and 1-2\% in case of isolated base building. This indicates that storey shear decreases with increase in storey height.

- In case of fixed base buildings the storey shear increases by $10-30 \%$ with addition of bracings and 30 $50 \%$ with addition of shearwalls.

- However in isolated base buildings the storey shear increases only by $5-15 \%$ with addition of bracings or shearwalls. This shows that storey shear reduces by isolated base.

\section{ACKNOWLEDGMENT}

I am greatly indebted to Mrs. SUMA DEVI Asst. Professor, Department of Civil Engineering SVCE Bangalore for her valuable guidance, sportive encouragement, much needed support and help in every needed sphere. Without her vision and versatility, this work would not have been feasible.

\section{REFERENCES}

[1] A. B. M. Saiful Islam, Mohammed Jameel, and Mohd Zamin Jumaat, "Seismic Isolation in Buildings to be a Practical Reality: Behavior of Structure and Installation Technique," Journal of Engineering and Technology Research, vol. 3, issue. 4, pp. 99-117, 2011.

[2] Computers and Structures Inc, CSI Analysis Reference Manual for Etabs, Berkeley, California, USA, 2005.

[3] C. V. R. Murty, "Earthquake Tips - Learning Earthquake Design and Construction," Department of Civil Engineering, Indian Institute of Technology Kanpur, 2005.

[4] Farzad Naeim, J. M. Kelly, "Design of Seismic Isolated Structures: From Theory to Practice," John Wiley and Sons Inc, 1999.

[5] IS 1893 Part-1, "Indian Standard Criteria for Earthquake Resistant Design of Structures, General Provisions and Buildings, (Fifth Revision)," Bureau of Indian Standards, New Delhi, 2002.

[6] C. Panayiotis Polycarpou, Petros Komodromos, "Earthquake-Induced Poundings of a Seismically Isolated Building with Adjacent Structures," Engineering Structures, vol. 32, pp. 1937-1951, 2010.

[7] Satish Nagarajaiah., Xiaohong Sun, "Base-Isolated FCC Building: Impact Response in Northridge Earthquake," Journal of Structural Engineering, vol. 127, issue. 9, pp. 1063-1075, 2001.

[8] S. K. Duggal, "Earthquake Resistant Design of Structures,” Oxford University Press, 2007. 


\section{BIOGRAPHIES}

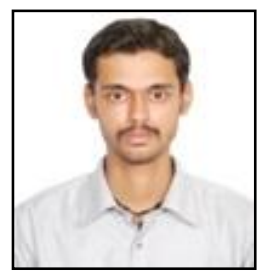

Raghunandan M H Assistant Professor at MSEC-Bangalore, obtained his MTech in Structural Engineering from SVCE-Bangalore and B.E. in Civil Engineering from SJBIT-Bangalore.

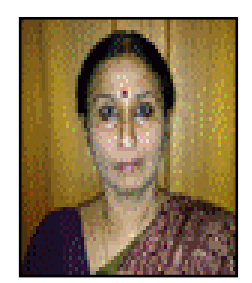

Suma Devi Assistant Professor at SVCEBangalore, obtained her M.E. in Earthquake Engineering from Bangalore University and B.Tech in Civil Engineering from Calicut University. She has 11 years industrial experience in residential, commercial, infrastructure projects \& 15 years of academic experience. 